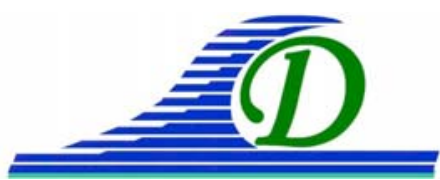

XIII ${ }^{\text {èmes }}$ Journées Nationales Génie Côtier - Génie Civil

Dunkerque, 2-4 juillet 2014

DOI:10.5150/jngcgc.2014.029 @ Editions Paralia CFL

disponible en ligne - $h$ ttp://www.paralia.fr - available online

\title{
L’évolution du trait de côte : un bon indicateur de la dynamique sédimentaire de l'avant côte ? Le cas du Languedoc-Roussillon
}

\author{
Cédric BRUNEL ${ }^{1}$, Raphaël CERTAIN ${ }^{1}$, Nicolas ROBIN ${ }^{1}$, Olivier RAYNAL ${ }^{1}$, \\ Nicolas ALEMAN ${ }^{1}$, Jean-Paul BARUSSEAU ${ }^{1}$
}

1. Laboratoire CEFREM, Université de Perpignan Via Domitia, 52 avenue Paul Alduy, 66860 Perpignan cedex 9, France.

cedric.brunel@univ-perp.fr

\section{Résumé :}

En milieu microtidal, peut-on considérer l'évolution du trait de côte comme un indicateur de référence de la dynamique sédimentaire sableuse ? La question est abordée dans ce travail par une analyse de la corrélation entre l'évolution du trait de côte et celle du bilan sédimentaire de l'avant-côte, à l'échelle séculaire (1895/1984/2009) et régionale (Languedoc-Roussillon). Les résultats montrent que les mouvements enregistrés au niveau de la ligne de rivage sont de moins en moins représentatifs du bilan sédimentaire (période 1895/1984 $\mathrm{r}^{2}$ : 0,31 ; période 1984/2009 $\mathrm{r}^{2}$ : 0,045). De 1984 à 2009, la tendance d'évolution du trait de côte et des fonds est même opposée pour plus d'un tiers du linéaire côtier étudié. Une discrétisation du littoral en secteurs dont la morphodynamique est dominée soit par l'évolution du trait de côte, soit par celle du bilan sédimentaire, soit marquée par une opposition entre ces deux indicateurs, révèle des ensembles spatiaux cohérents (lidos, embouchures de fleuves, secteurs aménagés, zone de convergence des dérives littorales). La morphodynamique de ces secteurs illustre les causes/effets des déconnexions qui peuvent exister entre le trait de côte et l'avant côte. Cette approche permet de proposer un modèle conceptuel à destination des chercheurs et gestionnaires qui analysent et gèrent le trait de côte.

Mots-clés : Ligne de rivage, Bilan sédimentaire, Long terme, Languedoc-Roussillon

\section{Introduction}

La connaissance de la dynamique sédimentaire des littoraux repose couramment sur l'analyse diachronique du trait de côte. En effet, comme l'évolution de la limite terre/mer traduit précisément en milieu micro-tidal le gain ou la perte de surface de plage émergée, elle est considérée comme un indicateur de référence de la dynamique sédimentaire côtière. De plus, l'évolution du trait de côte est relativement facile à obtenir par des levés de terrain, par des photographies aériennes ou de l’imagerie vidéo. Cependant, la pertinence de l'analyse de l'évolution du trait de côte repose sur l'hypothèse de l'existence d'une corrélation significative entre le bilan surfacique de la plage émergée et le sédimentaire de l'avant côte. Or, de nombreux facteurs naturels 


\section{Thème 2 - Dynamique sédimentaire}

(substrat affleurant, barres d'avant côte, apports fluviaux, transit sédimentaire, transport éolien), ou anthropiques (aménagements, rechargements), laissent supposer que la morphodynamique du prisme sableux n'est pas nécessairement homogène sur l'ensemble de son profil. L'objectif de ce travail est de confronter l'indicateur de référence couramment utilisé, l'évolution du trait de côte, à une vision plus globale de la dynamique du littoral fournie par les variations du volume sédimentaire disponible sur l'avant-côte (bilan sédimentaire). Tout d'abord, l'existence d'une corrélation significative entre ces deux indicateurs est étudiée et discutée. Ensuite, les écarts par rapport à la corrélation permettent de définir des secteurs aux comportements morphodynamiques types différents. Enfin, la spatialisation des comportements types éclaire le poids des facteurs dominants de contrôle du prisme sableux étudié.

\section{Contexte général}

La zone d'étude s'étend sur environ $200 \mathrm{~km}$ de littoral entre les villes d'Argelès/mer au sud et le Grau du Roi au Nord. Ce linéaire côtier, d'environnement micro-tidal, est constitué d'une succession de cordons littoraux sableux, entrecoupés par des caps rocheux (Cap Leucate, Cap d'Agde, Sète), qui délimitent quatre compartiments sédimentaires avec du sud au nord : le Roussillon, le Narbonnais, l'Hérault et le Golfe d'Aigues Mortes. Sous l'effet de la dérive littorale, estimée aujourd'hui entre 10000 et $40000 \mathrm{~m}^{3}$ /an selon les secteurs (DURAND, 1999 ; LNH/SOGREAH, 1984), les cordons littoraux ont isolé de nombreuses lagunes. Les sédiments qui composent les cordons littoraux proviennent principalement du Rhône et des fleuves côtiers: le Tech, la Têt, l'Agly, l'Aude, l'Orb et l'Hérault. A partir des années 60, le littoral du LanguedocRoussillon a été équipé d'aménagements côtiers avec la construction ex nihilo de 13 stations balnéaires construites autour de ports de plaisance. Aujourd'hui, plus de 30\% de la ligne côtière du Languedoc-Roussillon est équipée de structures de protection (IFEN, 2007) et depuis les années 1980-90, les gestionnaires du littoral procèdent localement à des rechargements artificiels en sables afin de compenser les phénomènes d'érosion du littoral.

\section{Méthodes et données}

Afin de déterminer et quantifier l'évolution du trait de côte et l'évolution du bilan sédimentaire à long-terme de l'avant-côte du Languedoc-Roussillon, trois levés de sondes bathymétriques $(1895 ; 1984$; 2009) et trois traits de côtes ont étés extraits de minutes de constructions, de cartes marines, de photographies aériennes, de levés GPS et LiDAR $(1895 ; 1984$; 2009). A partir de ces données, on a comparé entre deux périodes (1895/1984 et 1984/2009) l'avancée ou le recul du trait de côte (en m/an) aux volumes érodés ou sédimentés (en $\mathrm{m}^{3} / \mathrm{m} / \mathrm{an}$ ), mesurés entre le 0 et la profondeur de fermeture, au droit de 330 profils perpendiculaires au rivage et équidistants (densité $=1$ profil tous les $500 \mathrm{~m}$ ). En accord avec la marge d'erreur des sondes, une valeur de +/- 


\section{XIII ${ }^{\text {èmes }}$ Journées Nationales Génie Côtier - Génie Civil \\ Dunkerque, 2-4 juillet 2014}

$0.6 \mathrm{~m}^{3}$ a été retenue comme seuil à partir duquel les variations bathymétriques peuvent être considérées comme significatives. La plage aérienne n'est pas prise en compte dans les différentiels. Cependant les études précédentes ont montré que la plage aérienne ne représente qu'une part limitée du stock sédimentaire total du profil de plage (DURAND, 1999 ; CERTAIN, 2002).

L'analyse de la corrélation entre l'évolution du trait de côte et le bilan sédimentaire est faite pour deux périodes (1895/1984 et 1984/2009). Les profils sont ensuite discrétisés en 6 types de comportements (figure 3) qui se distinguent soit par l'importance de l'évolution du trait de côte ou du bilan sédimentaire, soit par l'opposition entre ces deux indices. Ainsi, (figures 1 et 2) on distingue des profils pour lesquels: (1) l'érosion de l'avant côte est plus importante que le recul du trait de côte (type nommé "érosif"), (2) le recul du trait de côte est plus important que l'érosion de l'avant côte (nommé "rétrogradant"), (3) l'accrétion de l'avant côte est plus importante que l'avancée du trait de côte (nommé "accumulant"), (4) l'avancée du trait de côte est plus importante que l'accrétion de l'avant côte (nommé "progradant"), (5) l'avancée du trait de côte est en opposition avec l'érosion de l'avant côte ("progradant-érosif"), (6) le recul du trait de côte est en opposition avec l'accrétion de l'avant côte ("rétrogradant-accumulant"). La spatialisation des types de tendances permet de définir des unités spatiales au comportement morphologique particulier et de proposer un modèle conceptuel (figure 3).

\section{Résultats/Discussion}

\subsection{Evolution du bilan sédimentaire et du trait de côte à long terme}

Durant la période 1895/1984, l'évolution du bilan sédimentaire et du trait de côte (figure 1) révèle de nettes différences entre les quatre compartiments sédimentaires du Languedoc-Roussillon. Spatialement, on distingue tout d'abord dans le Roussillon une évolution du trait de côte et du bilan sédimentaire en dent de scie, certainement en relation avec la succession de zones de nature différente: secteurs naturels, embouchures de fleuves et zones portuaires. Malgré cette alternance, le trait de côte et le bilan sédimentaire sont en moyenne en accrétion, avec 0,2 m/an d'avancée du trait de côte et $0,34 \mathrm{~m}^{3} / \mathrm{m} /$ an de gains au niveau de l'avant côte. Dans le Narbonnais, la partie située au sud de l'embouchure de l'Aude est globalement en accrétion avec 0,57 m/an d'avancée et $0,37 \mathrm{~m}^{3} / \mathrm{m} / \mathrm{an}$ de gains, mais pour la partie Nord du Narbonnais, de l'Aude au Cap d'Agde, l'érosion prédomine avec en moyenne $0,33 \mathrm{~m} / \mathrm{an}$ de recul et $0,13 \mathrm{~m} / \mathrm{m} / \mathrm{an}$ d'érosion des fonds. Plus au Nord, le linéaire côtier de l'Hérault est majoritairement en érosion. En effet, le lido de Sète recule en moyenne de 0,25 m/an et ses fonds perdent $0,28 \mathrm{~m}^{3} / \mathrm{m} / \mathrm{an}$ en moyenne. Seul le trait de côte prograde légèrement à l'extrémité sud du lido. Enfin, dans le Golfe d’Aigues Mortes, l'érosion prédomine avec des reculs maximums mesurés au droit du lido de Maguelone (-2 m/an). Dans le Golfe d’Aigues 


\section{Thème 2 - Dynamique sédimentaire}

Mortes quelques zones d'accrétion se situent directement en amont des ports de Palavas, Carnon, La Grande Motte et Port-Camargue. Pour la période 1984/2009, l'évolution du bilan sédimentaire et du trait de côte montrent un schéma généralisé: l'érosion de l'avant côte touche quasiment l'ensemble de la région (environ $-1 \mathrm{~m} / \mathrm{m} / \mathrm{an}$ ) alors que le trait de côte peut prograder encore (entre 0,5 et $2 \mathrm{~m} / \mathrm{an}$ ), là où le bilan sédimentaire est le moins négatif (localement dans le Roussillon, le sud du Narbonnais et le nord du Golfe d'Aigues Mortes).

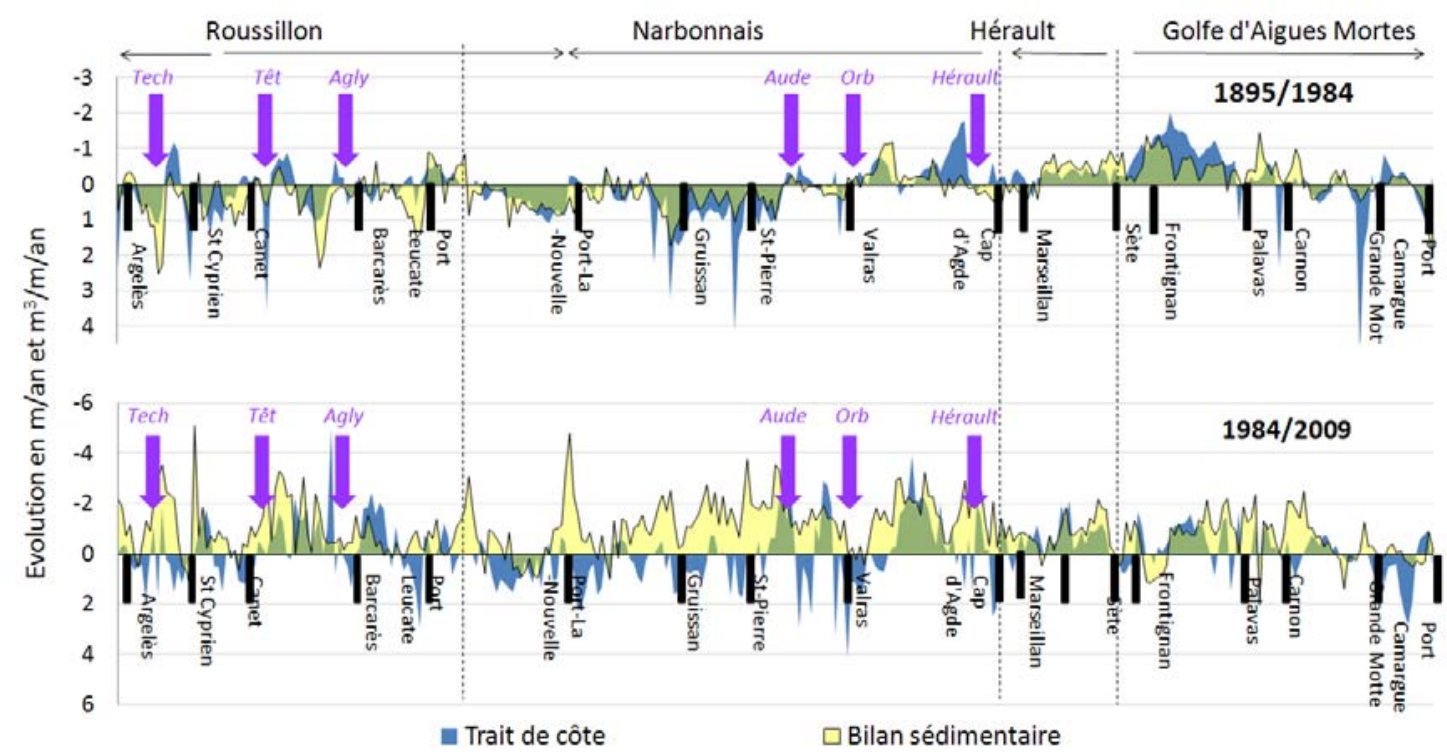

Figure 1. Evolution du trait de côte et du bilan sédimentaire en Languedoc-Roussillon de 1895 à 1984 (graphique du haut) et 1984 à 2009 (graphique du bas) par profil en $\mathrm{m} / \mathrm{an}$ et $\mathrm{m}^{3} / \mathrm{m} / \mathrm{an}$. Localisation des compartiments sédimentaires et direction des dérives littorales, embouchures des fleuves et principaux ports.

4.2 Analyse du rapport entre l'évolution du bilan sédimentaire et celle du trait de côte Il existe une corrélation entre l'évolution du trait de côte et le bilan sédimentaire de l'avant côte pour la période 1895/1984 $\left(\mathrm{r}^{2}: 0,31\right)$ et aucune corrélation pour la période $1984 / 2009\left(r^{2}: 0,045\right)$ (figure $\left.2, n=330\right)$. Pour la première période, l'évolution du trait de côte est représentative de l'évolution des fonds pour les profils distribués le long de la droite de régression. Selon l'équation de la droite, $1 \mathrm{~m} / \mathrm{an}$ d'avancée du trait de côte correspondrait à $0.31 \mathrm{~m}^{3} / \mathrm{m} / \mathrm{an}$ de gains sédimentaires au niveau de l'avant côte et inversement. Toutefois les profils distribués le long de la droite (en considérant une marge de $+/-10 \mathrm{~m}$ pour les traits de côte et $+/-0,6 \mathrm{~m}^{3}$ pour le bilan sédimentaire) ne représentent qu'un cinquième environ du linéaire côtier étudié. Durant la deuxième période, l'absence de corrélation illustre le basculement de la dynamique du système se traduisant par une l'érosion quasi générale de l'avant côte (BRUNEL et al., 2014) malgré un trait de côte qui peut continuer à prograder (figure 1). Ce résultat limite 


\section{XIII ${ }^{\text {èmes }}$ Journées Nationales Génie Côtier - Génie Civil \\ Dunkerque, 2-4 juillet 2014}

l'utilisation de cet outil statistique pour la période récente ainsi que les comparaisons entre des deux périodes.
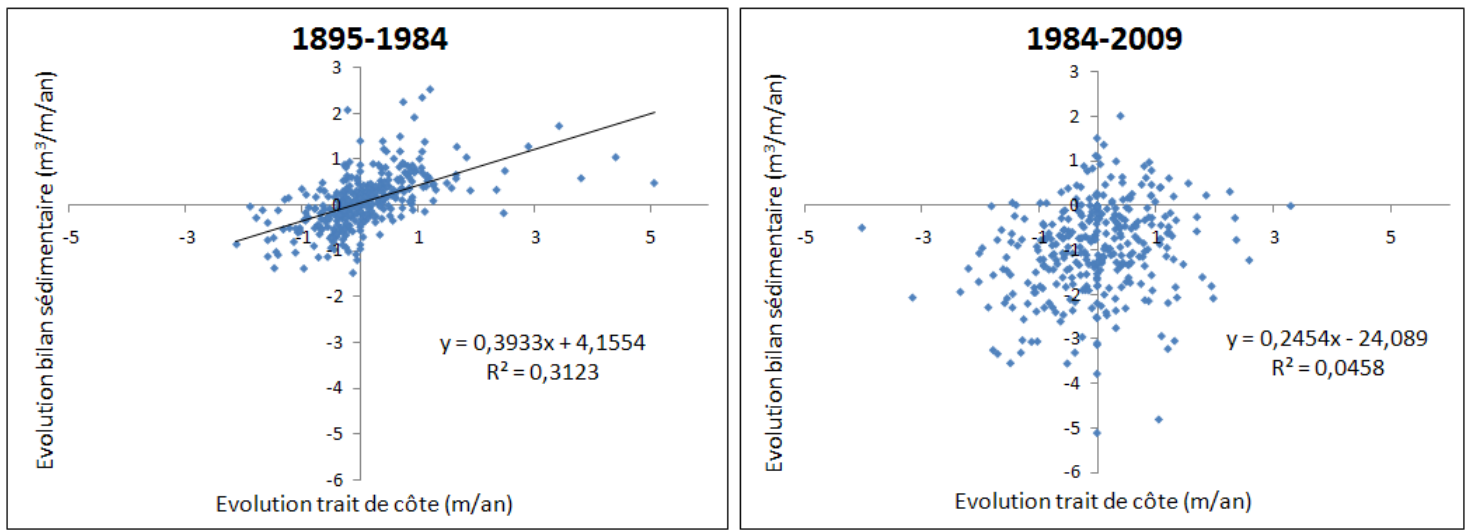

Figure 2. Graphiques de corrélation entre les évolutions du trait de côte et du bilan sédimentaire.

Néanmoins, la large distribution des points dans les graphiques de corrélations permet de distinguer 6 différents types de comportements du littoral. Le calcul de la moyenne de l'évolution du trait de côte et du bilan sédimentaire pour chacun de ces types révèle les écarts significatifs qui existent entre eux (Tableau 1). Par exemple de 1984 à 2009, le type "progradant" gagne en moyenne $0,9 \mathrm{~m} / \mathrm{an}$ d'avancée du trait de côte pour 0,32 $\mathrm{m}^{3} / \mathrm{m} /$ an gagnés sur l'avant côte. Cela représente 3 fois plus de surface de plage gagnée par rapport au type "accumulant" qui gagne en moyenne 0,28 m/an au niveau du trait de côte pour $0,96 \mathrm{~m}^{3} / \mathrm{m} /$ an gagnés en mer. Parallèlement, le type "accumulant" gagne 3 fois et demie plus de sédiments sur l'avant côte que le type "progradant". On constate que les écarts sont tout aussi importants entre tous les autres types identifiés (Tableau 1). La typologie définie ici repose donc sur des ordres de grandeurs significatifs qui illustrent les forts décalages et les oppositions qui existent entre l'évolution du trait de côte et celle des fonds en Languedoc-Roussillon.

Les types identifiés correspondent à des unités géomorphologiques homogènes caractérisés par des morphodynamiques propres qui illustrent les causes/effets des déconnexions progressives trait de côte/bilan sédimentaire. L’évolution séculaire de ces unités géomorphologiques et anthropiques peut être synthétisée dans un schéma conceptuel (figure 3). Par exemple, le lido de Maguelone montre un caractère exclusivement "rétrogradant" probablement en relation avec une dynamique naturelle de "roll over". En effet, des phénomènes d’overwash, caractérisés par des cônes de débordement en arrière cordon, sont observables sur ce lido étroit, naturel et de faible altitude. A l'inverse, le lido entre Sète et Marseillan montre un caractère exclusivement "érosif". La présence de voies de communications ainsi que l'altitude du lido (environ $4 \mathrm{~m}$ ) fige la dynamique de "roll over" et favorise probablement l'érosion des fonds. Le 


\section{Thème 2 - Dynamique sédimentaire}

centre du Narbonnais est "progradant-érosif", ce qui reflète les effets du transport éolien de la plage vers l'avant côte dans un secteur influencé fortement par les vents de terre (Tramontane), ainsi qu'un mouvement transversal des sédiments vers la côte via un régime de houles frontales dominantes. Cela traduit aussi la diminution du volume des barres d'avant côte du Narbonnais, de moins en moins alimentées par les zones sources. En dehors des profils naturels du Narbonnais, les profils de type "progradant-érosif" se situent en amont des jetées des ports et embouchures. En bloquant une partie de la dérive littorale elles font prograder la ligne de rivage sans pour autant faire gagner du sable à l'avant côte. Enfin, la majorité des secteurs équipés d'épis et d'enrochements sont "érosifs". Excepté les brises lames qui font localement prograder le trait de côte dans la zone d'abri qu'ils créent, les aménagements ralentissent globalement le recul du trait de côte sans toutefois contrecarrer l'érosion de l'avant côte. Enfin, aucun comportement distinct n'a été identifié pour les secteurs qui ont fait l'objet de rechargements artificiels, l'échelle de temps de l'étude étant mal adaptée pour distinguer ce type d'intervention.

Tableau 1. Moyenne et représentation numérique des valeurs d'évolution du trait de côte et du bilan sédimentaire par types de comportements identifiés.

\begin{tabular}{llcccc}
\hline Type de comportement & $\begin{array}{c}\text { TDC } \\
(\mathbf{m} / \mathbf{a n})\end{array}$ & $\begin{array}{c}\text { Bilan } \\
\left(\mathbf{m}^{3} / \mathbf{m} / \mathbf{a n}\right)\end{array}$ & $\begin{array}{c}\text { Profils } \\
(\text { Nbre.) }\end{array}$ & $\begin{array}{c}\text { Linéaire } \\
\text { côtier (\%) }\end{array}$ \\
\hline $\mathbf{1 8 9 5 / 1 9 8 4}$ & 0,32 & 0,68 & 57 & 17 \\
& Accumulant & 1,02 & 0,43 & 82 & 25 \\
& Progradant & $-0,28$ & $-0,63$ & 51 & 15 \\
& Erosif & $-0,79$ & $-0,35$ & 66 & 20 \\
& Rétrogradant & $-0,34$ & 0,35 & 45 & 14 \\
& Rétrogradant-accumulant & 0,40 & $-0,22$ & 29 & 9 \\
& Progradant-érosif & & & & \\
& & 0,28 & 0,90 & 14 & 4 \\
$\mathbf{1 9 8 4 / 2 0 0 9}$ & Accumulant & 0,96 & 0,32 & 19 & 6 \\
& Progradant & $-0,52$ & $-1,62$ & 117 & 35 \\
& Erosif & $-1,19$ & $-0,77$ & 39 & 12 \\
& Rétrogradant & $-0,43$ & 0,33 & 19 & 6 \\
& Rétrogradant-accumulant & 0,66 & $-1,05$ & 121 & 37 \\
\hline
\end{tabular}




\section{XIII ${ }^{\text {èmes }}$ Journées Nationales Génie Côtier - Génie Civil \\ Dunkerque, 2-4 juillet 2014}

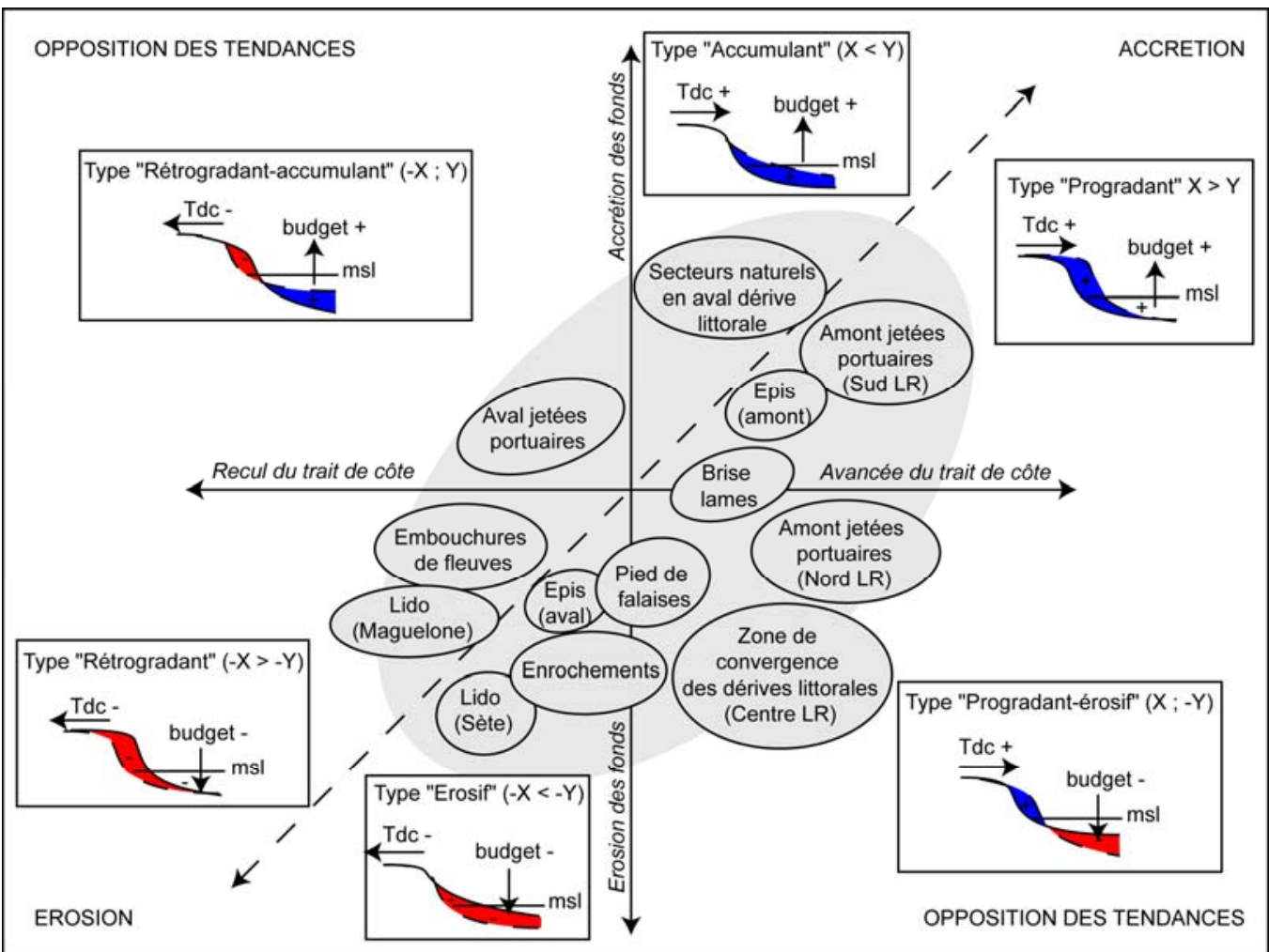

Figure 3. Schéma conceptuel de la relation entre le trait de côte et le bilan sédimentaire par unités morphologiques et anthropiques en Languedoc-Roussillon (1895/2009).

\section{Conclusions}

En Languedoc-Roussillon, les mouvements enregistrés au niveau de la ligne de rivage ne sont pas toujours représentatifs de l'ensemble des mouvements de l'enveloppe du prisme sableux, en particulier depuis 1984. Ces observations sont probablement en relation avec un bilan sédimentaire négatif à l'échelle de la région et une anthropisation croissante perturbant la dynamique naturelle du système. Les écarts qui existent entre les mouvements du trait de côte et ceux des fonds peuvent approcher un facteur 3. Ce constat est d'autant plus marquant que pour un quart (1895/1984) puis un tiers (1984/2009) du linéaire côtier de la région les tendances d'évolution du trait de côte et des fonds sont opposées. Ces constats remettent en cause l'application de formules simples destinées à définir des bilans sédimentaires à partir de valeurs de variation du rivage (CERC, 1984; JIMENEZ \& SANCHEZ-ARCILLA, 1993) et l'utilisation de modèles qui reposent sur le concept de profil d'équilibre (GENESIS, S-BEACH) (LARSON \& KRAUS, 1989), les profils n'évoluant pas à pente constante. En perspective d'analyse, la prise en compte de la géométrie fine du prisme sableux via une campagne de sismique haute résolution à l'échelle de la région (en cours d'analyse) devrait grandement éclairer les facteurs géologiques de contrôle du littoral, comme le rôle de l'épaisseur du prisme sableux ou l'influence du substrat affleurant localement. 


\section{Remerciements}

Les auteurs remercient la Direction régionale de l'Environnement, de l'Aménagement et du Logement du Languedoc-Roussillon (DREAL LR) pour sa contribution à cette étude.

\section{Références bibliographiques}

BRUNEL C., CERTAIN R.., SABATIER F., ROBIN N., BARUSSEAU J.P., ALEMAN N., RAYNAL O. (2014). 20th century sediment budget trends on the Western Gulf of Lions shoreface (France): An application of an integrated method for the study of sediment coastal reservoirs. Geomorphology, Vol. 204, pp 625-637. http://dx.doi.org/10.1016/j.geomorph.2013.09.009

CERC (1984). Shore Protection Manual. Volumes I et II, Department of the Army, US Army Corps of Engineers.

CERTAIN R. (2002). Morphodynamique d'une côte sableuse microtidale à barres : le Golfe du Lion. Thèse de Doctorat, Université de Perpignan, 209 p.

DOLAN R., HAYDEN B.P., MAY P., MAY S. (1980). The reliability of shoreline change measurements from aerial photographs, Shore and Beach, Vol. 48(4), pp 22-29.

DURAND P. (1999). Cinématique d'un littoral sableux à partir de photographies aériennes et de cartes topographiques. Géomorphologie: relief, processus, environnement, $\mathrm{n}^{\circ} 2$, pp 155-166.

IFEN (2007). Institut Français de l'environnement $n^{\circ} 120,4$ p.

JIMENEZ J.A. SANCHEZ-ARCILLIA A. (1993). Medium-term coastal response at the Ebro delta, Spain. Marine Geology, Vol. 114, pp 105-118. http://dx.doi.org/10.1016/0025$\underline{3227(93) 90042-\mathrm{T}}$

LARSON M., KRAUS N.C. (1989). SBEACH : Numerical model for simulating storm induced beach change, Report 2, US Army Corps of Engineers.

LNH/SOGREAH (1984). Catalogue sédimentologique des côtes françaises. Côtes de la Méditerranée. Collection de la Direction des Etudes et Recherches d'Electricité de France, №52 Eyrolles, 426 p. 